\title{
A Long-Period Pi 2 Associated with a Mini-Substorm Observed by the Japanese Satellite JIKIKEN (EXOS-B)
}

\author{
T. Saito, ${ }^{*}$ K. Yumoto, ${ }^{*}$ I. Aoyama, ${ }^{* *}$ F. Toyama, ${ }^{* *}$ T. SakuraI, ${ }^{* *}$ \\ T. TAKAHASHI, ${ }^{* *}$ and Y. TonegaWA** \\ * Onagawa Magnetic Observatory and Geophysical Institute, \\ Tohoku University, Sendai, Japan \\ ** Laboratory of Space Science, Tokai University, Tokyo, Japan
}

(Received October 27, 1980)

Being associated with a typical mini-substorm, a very-long-period Pi 2 event was observed simultaneously at both Onagawa, Japan, and the satellite, JIKIKEN, at 14-15 hUT on December 11, 1978. The fundamental period of the Pi 2 attained to $220 \mathrm{sec}$ and the range of the substorm at Onagawa on the $23 \mathrm{~h}$ local time meridian was only $2 \mathrm{nT}$. The plasmapause was observed by JIKIKEN on the $21 \mathrm{~h}$ local time meridian to be at $L>6.3$.

This Pi 2 event in the extreme case is well explained by such an odd-mode model that a temporal odd-mode torsional oscillation of the dipole-like field lines anchored in the auroral ovals is observed as Pi 2. The analysis reveals that the ignition region for this mini-substorm must be $|X|>16 R_{\mathrm{E}}$ in the magnetotail and that the tail-like configuration of the field line must be changed to the dipole-like one within several min. This event provides valuable information on one of the smallest cases of the magnetospheric substorm.

\section{Introduction}

It is quite important for the study of magnetospheric substorm to find the simplest and the most fundamental substorm and to investigate the occurrence mechanism of it. The simplest one was denot:d by SAITo et al. (1976) as minisubstorm based on various kinds of ground-based magnetograms. The purpose of the present study is to analyze the first mini-substorm that has ever been observed by satellites and to interpret its mechanism in relation to the plasmasheet model on Pi 2 type magnetic pulsation. As for the magnetometer and orbital elements of JIKIKEN, the reader may refer to AoYAMA et al. (1979).

\section{Observation}

Figure 1A shows the mini-substrom event observed on December 11, 1978 while the satellite "JIKIKEN" was in the night-side magnetosphere. Geomagnetic 

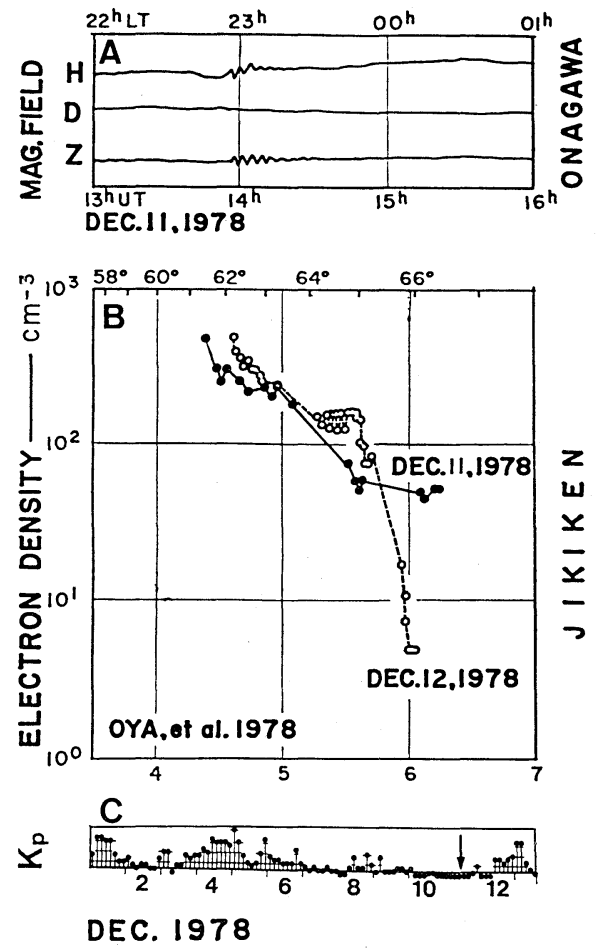

Fig. 1. Mini-substorm event on Dec. 11, 1978
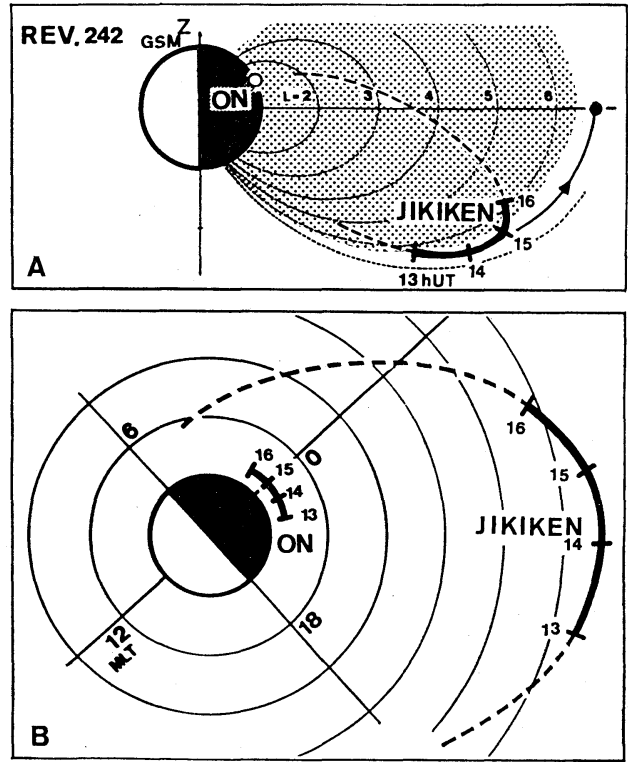

Fig. 2. Trajectory during the mini-substorm. 
activity had been extremely low for about 10 days before the event as shown in $K_{p}$ in Fig. 1C. The stimulated plasma wave (SPW) experiment at JIKIKEN revealed that the plasmapause was situated at $L>6.3$ before the event, while $L=5.6$ after the event (OYA, 1979). Both of Figs. 1B and 1C confirm that the event is a typical minisubstorm and support out assertion that mini-substorm occurs during an extremely calm magnetic condition.

The trajectory of JIKIKEN is shown in Fig. 2, where the equatorial position of the field line anchoring in Onagawa is also exhibited. The position of JIKIKEN is also projected on the equatorial plane in Fig. $2 \mathrm{~B}$ along the dipole field line. It is noteworthy that both of JIKIKEN and Onagawa were situated in the midnight sector where substorms are well registered.

\section{Analysis}

The magnetic field variations observed at both JIKIKEN and Onagawa during the mini-substorm event are analyzed into hissagrams as shown in Fig. 3. (As for hissagram, see SAITO, 1976). It is revealed from Fig. 3 that the event observed at Onagawa contains two spectral components at $\sim 220$ and $\sim 140 \mathrm{sec}$, respectively. The $\sim 140 \mathrm{sec}$ component of the mini-substorm is also recognized clearly in the

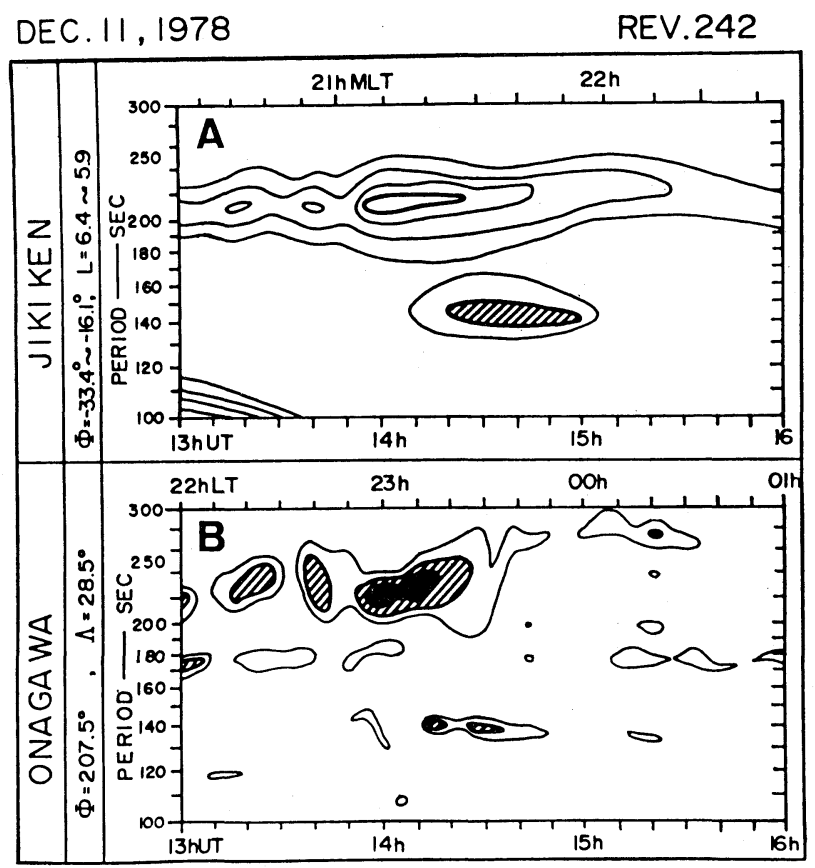

Fig. 3. Hissagram of the mini-substorm. 
dynamic spectrogram obtained from the JIKIKEN magnetometer. (It should be noted that a fluctuating altitude of the satellite due to the 102-m (tip-to-tip) antennas generates a fluctuating magnetic noise near around $210 \mathrm{sec}$ as shown in Fig. 3A.)

\section{Interpretation}

All substorm is surely associated with Pi-type magnetic pulsation (SAITo, 1969). There is an inverse relation between the Pi 2 period and the substorm magnitude; substorm with the smaller magnitude is associated with Pi 2 with the longer period (SAITo et al., 1968; SAIto et al., 1976). According to the odd-mode model of Pi 2, it has generally two spectral component; one correspond to the temperal odd-mode oscillation of the field line anchoring in the midnight auroral oval (SAITO, 1979; KUWASHIMA et al., 1979), and the other to the field line neat the plasmapause (FUKUNISHI, 1975). The $\sim 220 \mathrm{sec}$ component of the mini-substorm is considered to be the former, while the $\sim 140 \mathrm{sec}$ component belongs to the latter. It should be noted here that JIKIKEN was situated near the plasmapause while it observed the $\sim 140 \mathrm{sec}$ component of magnetic fluctuations.

\section{Calculation}

The odd-mode model is schematically illustrated in Fig. 4A. Here we commit ourselves to the reconnection model for the triggering of the substorms where Alfvén waves generated at the $X$-type neutral line in the magnetotail (0) are considered to

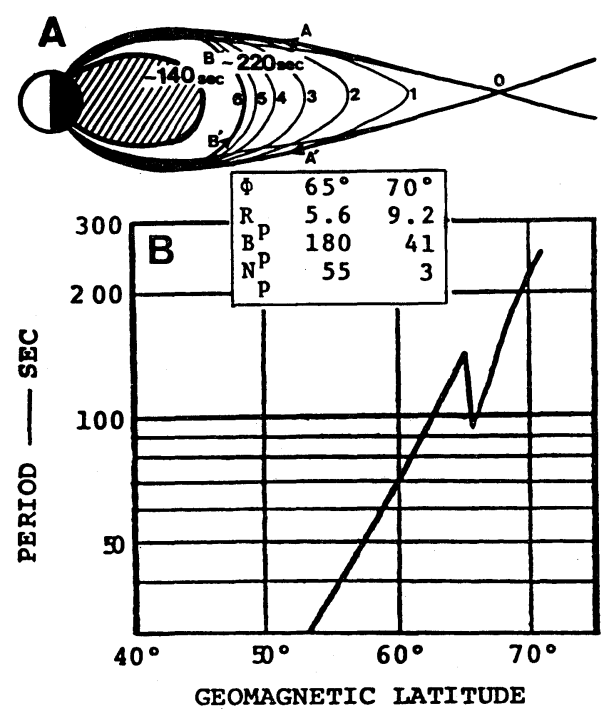

Fig. 4. Odd-mode model of Pi 2. 
propagate along the field lines $A$ and $A^{\prime}$, being reflected back by the polar ionosphere, and make a temporal standing oscillation along the field line $B B^{\prime}$. If radial distance, magnetic field intensity $\left(B_{\mathrm{P}}\right)$, and plasma density $\left(N_{\mathrm{P}}\right)$ of the apex $\left(R_{\mathrm{P}}\right)$ of a magnetic field line anchoring in geomagnetic latitude $(\Phi)$ are assumed to be $\Phi=$ $70^{\circ}, R_{\mathrm{P}}=9.2, B_{\mathrm{P}}=41, \mathrm{~N}_{\mathrm{P}}=3$ for the field line $B B^{\prime}$ for the mini-substorm event as listed at the middle of Fig. 4, the period of the odd-mode oscillation is calculated to be $220 \mathrm{sec}$ as shown in Fig. 4B. On the other hand we can assume from the SPW experiment at JIKIKEN that $\Phi=65^{\circ}, R_{\mathrm{P}}=5.6, B_{\mathrm{P}}=180$, and $N_{\mathrm{P}}=55$ for the field line just inside the plasmapause, where a temporally standing oscillation is secondarily excited due to the plasma instability at the $X$-type neutral line. The oscillation near the plasmapause is then calculated to be $140 \mathrm{sec}$ as shown in Fig. 4B. The calculated values of 220 and $140 \mathrm{sec}$ correspond quite well with the observed values and justify the odd-mode model (namely, the plasma-sheet model (SAITO et al., 1970) of Pi 2.).

\section{Discussion and conclusion}

A mini-substorm event was observed simultaneously at the satellite, JIKIKEN, and at the ground-based station, Onagawa, at 14h UT on 11th of December, 1978. A comparative dynamic spectral analysis of these data provides the following conclusions on the wave phenomenon during the mini-substorm.

1) The $\sim 220$-sec component which was observed at Onagawa is the fundamental odd mode of the Alfvén wave that was propagated from the plasma instability source at the $X$-type neutral line in the magnetotail which resulted the substorm event.

2) The $\sim 140$-sec component which was observed commonly at JIKIKEN and at Onagawa is the Alfvén wave excited secondarily on the plasmapause by the plasma instability in the magnetotail.

3) The present analysis substantiates the plasma-sheet theory (SAIto et al., 1970) that the main component of Pi 2 is related to the field line corresponding not to the plasma pause, but to the plasma sheet.

4) The present Pi 2 event has so clear and long period that can be well detected on ordinary magnetograms. For example, this event is clearly identified as Pi 2 wave with as large amplitude as $4 \mathrm{nT}$ on the oridnary magnetogram from Kiruna even though it was situated near the noon meridian during the event. It means that $\mathrm{Pi} 2$ associating with mini-substorm is quite useful to study the world-wide characteristics of Pi 2 waves.

Besides the Pi 2 waves, the present analysis of the Dec. 11, 1978 mini-substorm event derived the following important informations on the magnetosphere physics. (The reader may refer to SAITo et al. (1979) for the further explanation on the derivation.) 
1) The position of the $X$-type neutral line (0 in Fig. 4A) must be $X \sim-20 R_{\mathrm{E}}$ for the smallest substorm, while $X \sim-5 R_{\mathrm{E}}$ for the largest substorm.

2) Time duration of the contraction from the tail-like magnetic field configuration to the dipole-like one (see Fig. 4A) must be $\sim 4$ min for the smallest substorm while $\sim 0.5 \mathrm{~min}$ for the largest substorm.

3) Although the plasmasphere was considered to need about one month to expand up to $\sim 6 R_{E}$, the present analysis reveals that it needs only about one week. This conclusion agrees well with the SPW experiment.

\section{REFERENCES}

Aoyama, I., F. Toyama, T. Takahashi, Y. Tonegawa, T. Sakurai, T. Saito, and K. Yumoto, The field-aligned currents observed by "JIKIKEN"., Proc. Japanese IMS Symp., held at ISAS, Tokyo Univ. on 12-14, Nov., 126-132, 1979.

FukUNISHI, H., Polarization changes of geomagnetic Pi 2 pulsations associated with the plasmapause, J, Geophys. Res., 80, 98-110, 1975.

Kuwashima, M. and T. Saito, Spectral characteristics of magnetic Pi 2 pulsations in the auroral region and lower latitudes, J. Geophys. Res., 1979 (submitted).

OYA, H., Summary on plasma wave emissions observed by Jikiken-preliminary report for the initial phase of the observation results, Magmetospheric Study 1979, 200-213, 1979.

SaIto, T. and S. Matsushita, Solar cycle effects on geomagnetic Pi 2 pulsations, J. Geophys. Res., 73, 267-286, 1968.

SAIto, T., Geomagnetic pulsations, Space Sci. Rev., 10, 319-412, 1969.

SAITo, T., The HISSA (high-speed spectrum analysis) method to analyze various time-varying phenomena in space physics, Rep. Ionos. Space Res. Jpn., 30, 3/4, 69-80, 1976.

SAIto, T., $N$-type reconnection model to interpret the mechanism of mini-substorm, J. Atmos. Terr. Phys., 1979 (submitted).

Saito, T., K. Yumoto, I. Aoyama, F. Toyama, and T. TAKahashi, Typical mini-substorm and longperiod Pi 2 event observed by Jikiken satellite on December 11, 1978, Bull. Inst. Space Aeron. Sci., Univ. Tokyo, 1979 (in press).

SAIto, T. and T. SAKURAI, Mechanism of geomagnetic Pi 2 pulsations in magnetically quiet condition, Sci. Rept. Tohoku Univ., Ser. 5, Geophys., 20, 49-70, 1970.

Saito, T., T. SakuraI, and Y. Koyama, Mechanism of association between Pi 2 pulsation and magnetospheric substorm, J. Atmos. Terr. Phys., 38, 1256-1277, 1976. 